
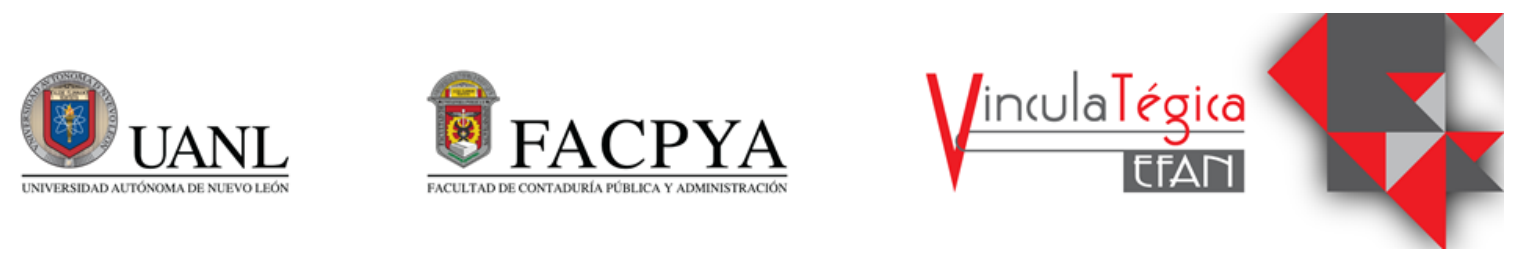

\title{
Banca Ética en México, fomento al emprendimiento social
}

\author{
Juan Antonio Molina Garnica ${ }^{1}$ y Yadira Zulith Flores Anaya ${ }^{2}$ \\ ${ }^{1}$ Universidad Autónoma de Baja California, amolina33@uabc.edu.mx, parque internacional industrial Tijuana, \\ 22427,Tijuana B.C., (646)189-50-45. \\ ${ }^{2}$ Universidad Autónoma de Baja California, flores.yadira@uabc.edu , parque internacional industrial Tijuana, \\ 22427,Tijuana B.C., (664)
}

Información del artículo revisado por pares

Fecha de aceptación: junio-2021

Fecha de publicación en línea: diciembre-2021

DOI: https://doi.org/10.29105/vtga7.1-111

\section{Resumen}

Con la evolución de la economía, es realmente necesario ver nuevos métodos de financiamiento, especialmente en los negocios sociales después del COVID- 19; la pandemia global que atacó a las personas en salud, trabajos y dinero. El Banco Ético es la nueva propuesta para los negocios sociales, buscando el equilibrio entre el comercio y la responsabilidad social. El objetivo de este trabajo es mostrar que tan necesario es el Banco Ético en México, para ayudar al gremio microempresarial, promoviendo la inversión social, contrario a la banca tradicional, que solo busca ganancias, y, si el negocio no las demuestra, el banco rechaza el financiamiento. La razón más común en México para negar el financiamiento es la falta de historial crediticio, esto es irracional, debido a que el propio banco no aprueba el crédito que permitirá tener el historial.

Palabras claves: Banco ético, Financiamiento, Negocio Social, Responsabilidad Social.

\begin{abstract}
With the changing evolution of the economy, a new financing method is necessary, especially in social business after covid 19, the global pandemic affected people in many areas such as health, work and money. The ethical bank is the new proposal for business, finding the balance between trade and social responsibility. The objective of this paper is to show how necessary is the ethical bank in Mexico to help business unions and promoting the social invest, in contrast with the traditional bank, that only want profit and if business projects request financing but if don't show benefit for the bank, they're rejected. Lack of credit history is the most common reason for rejecting financing in Mexico, which is irrational, because if the bank doesn't approve the credit, it doesn't allow the credit history to be created.
\end{abstract}

Keywords: Ethical Bank, Financing, Social Business, Social Responsibility.

JEL: M14-N20-P33-P36 


\section{I.- INTRODUCCIÓN}

Hoy en día, obtener un crédito es sencillo, este se utiliza para apalancar las necesidades de las personas o las empresas, sírvase como activo circulante, inversión inicial o liquidación de deudas. El crédito ayuda a financiar proyectos, y es fácil obtenerlo, siempre y cuando se cumplan con ciertos requisitos.

Incumplir con los requisitos documentales requeridos por el banco, ocasiona que el financiamiento pueda ser negado, entre estos documentos se encuentran; el historial crediticio, el comprobante de domicilio, y el comprobante de ingresos, entre otros.

Esto puede sonar lógico o razonable, hasta cierto punto, ya que la falta de algunos de estos documentos impide el poder obtener préstamos por parte del banco, debido a que no existe una garantía de por medio.

Pero, qué pasa cuando el negocio apenas está iniciando. En una economía donde el desempleo va al alza, el emprendimiento es la solución, pero para iniciar un negocio es necesario contar con un capital inicial, invertir dinero, con el que no se cuenta en su mayoría.

Es aquí donde los negocios sociales se han visto limitados; emprendedores con ideas de negocios, que no solo generan empleo, sino que también buscan resolver alguna problemática socio ambiental.

Es así que, el sistema financiero tradicional que actualmente opera en México, no puede colaborar en la creación de negocios sociales, hablando de aquellos de nueva creación. Por lo tanto, la presente investigación propone al Banco Ético como una solución.

La hipótesis de este documento radica en el supuesto de que la Banca Ética, incentiva el financiamiento formal para los negocios sociales con el fin de apoyar a la sociedad, más que para buscar beneficios propios, siendo consciente de las necesidades y problemáticas que estos tienen para iniciar operaciones.

\section{II.- MARCO TEÓRICO.}

Los antecedentes de la investigación indican que la idea de la Banca Ética nació a partir de la necesidad de dar solución a un problema de financiamiento al otro lado del mundo, específicamente en Bangladesh. Es así que, a partir del trabajo del profesor Muhammad Yunus, quien realizó el primer préstamo con intención social, sustentado con capital propio, con la finalidad de que otras personas pudieran producir, con un pequeño financiamiento (Peruzzo, 2020).

Es así que el profesor Yunus, busca resolver problemas socio ambientales, esto, junto a su trabajo, le otorgó el mérito al premio nobel de la paz en 2006. En su visita a México, él expresó la necesidad de apoyar a estas causas, y también de la ventaja con la que las nuevas generaciones cuentan para emprender, debido a las tecnologías (Pineda, 2019).

De esta manera, es que a través de otorgar créditos en pequeñas cantidades y comisiones inferiores a las del sistema financiero convencional, Yunus conforma Grameen Bank, busca apoyar las causas sociales, sin perjudicar el medio ambiente, todo esto tras un pequeño préstamo de un dólar, a baja tasa de interés, para una mujer artesana que elabora asientos de bambú(Grameen Bank, 2021).

Es así como empiezan a surgir los negocios sociales; a través de la idea del Profesor Yunus. Peruzzo (2020) define a la Banca Ética como el"conjunto de entidades financieras que basan su accionar en valores socialmente aceptados, en el bienestar social y cuidado del medioambiente; dejando de lado la especulación y la obtención de la máxima rentabilidad como objetivo principal" (p.?)

De lo anterior, se comprende que al contrario de la banca tradicional, el sistema de Banca Ética, busca generar un doble impacto, financiando a empresas, con enfoque social que a su vez, buscan subsanar una necesidad social y/o ambiental.

Con base en estas características y motivados por el profesor Yunus, nace Triodos Bank, el pionero de la banca ética. Este banco fue fundado en los países bajos en el año de 1980, si bien, la concepción de lo que luego se convertiría en Triodos Bank surgió desde 1968, su constitución legal no sucede sino hasta 1971, con ideas de varios profesionistas como Adriaan Deking Dura, Dieter 
Brüll, Lex Bos y Rudolf Mees quienes buscaban cómo gestionar el dinero de forma sostenible (Triodos Bank UK, 2021).

Es así como Triodos Bank UK se enfoca en el apoyo de personas y empresas que beneficien a la sociedad y el medio ambiente, dirigiéndose al ámbito ecológico, cultural y social, negando así préstamos a empresas que anteponen las utilidades a las personas mismas (Ídem, 2021).

Estos enfoques son los que le dan vida a la banca ética: anteponer el beneficio de la sociedad, ya sea en cuestiones sociales, culturales o medioambientales, inclusive la combinación de cualquiera de estas, antes que las ganancias o la riqueza; y sobre todo, la importancia de saber en qué se está invirtiendo el dinero, y conocer los beneficios que traerá.

Beneficios tales como, menor gasto de agua, reciclar plásticos, alimentar a personas con necesidad, son algunos de los giros que la banca ética busca financiar, no importando las utilidades que estas generen, si no la aportación social que realizan.

En este sentido Melé (2018) explica que la banca ética, es una idea simple, con base en el sentido común de una reacción humanitaria, es así que este modelo de finanzas éticas nace como respuesta a eventos como la esclavitud, la guerra de Vietnam, el régimen del apartheid, entre otros.

Evitando de esta manera, que las inversiones que los clientes de un banco aportan, terminen en el financiamiento de un régimen autoritario, guerra, o cualquier amenaza a los derechos humanos y a la destrucción del ecosistema.

La definición de Peruzzo (2020) y los ideales que ayudaron a establecer Triodos Bank concuerdan satisfactoriamente, por lo que es importante establecer que las necesidades sociales y climáticas, son los pilares para que la banca ética pueda existir, es por ello que ha adquirido cada vez mayor presencia en el resto del mundo.

En 2003, Europa, específicamente Italia y España, con la fundación Fiare, cuando se une con la Banca Polare Ética y recuperan el capital invertido, es entonces que se funda la banca ética Fiare, esto durante el año 2014 (Fiare Banca Ética, 2021).
Para 2017, Chile inicia con el modelo de banca ética, con el objetivo de apoyar a distintas organizaciones con beneficios sociales y necesidad de financiamiento (Cantuarias, 2021), esto siembra las bases para que otros países de Latinoamérica se unan al objetivo.

Uno de los ejemplos más cercanos a la banca ética, actualmente, es la empresa Doble Impacto, una plataforma de inversión en vías de convertirse en banca ética para 2022. Su misión, como su nombre lo indica, es generar un impacto doble en las inversiones, apoyando tanto a empresas e instituciones que beneficien en lo económico, social y cultural (Doble Impacto, 2021).

Doble Impacto (2021), se enfoca en invertir en educación de calidad, industria creativa y turismo sustentable, realizando evaluaciones integrales de las empresas que solicitan financiamiento con ellos, aunado a esto, cuentan con un grupo de especialistas que gestiona un préstamo responsable.

En los últimos años, la banca ética se ha enfocado en tres sectores económicos específicos:, educación y cultura, desarrollo social y medio ambiente. cuenta con influencia en Santiago de Chile, Montevideo, Uruguay, Buenos Aires en Argentina y São Paulo, Brasil, con intención de llegar a Bogotá, Colombia y Ciudad de México, México (Banca Ética Latinoamérica, 2021).

Según cifras de Banca Ética Latinoamérica (2021), se han invertido alrededor de $\$ 10,167,000$ dólares, en educación y cultura; también se han invertido $\$ 12,926,000.00$ dólares en desarrollo social y $\$ 8,651,000.00$ dólares en medio ambiente, demostrando así la necesidad de este tipo de instituciones.

Es importante mencionar que en los países latinoamericanos que han establecido el modelo de banca ética, han comenzado a través de fundaciones, las cuales se dirigían a fondos de impacto social.

De lo anterior, es importante mencionar, que hasta el momento México no cuenta con una banca ética, si bien existen instituciones financieras, estas no contemplan un objetivo social.

Esto dificulta el emprendimiento social en el país, debido a que el financiamiento se encuentra sujeto a las políticas del sistema financiero formal, 
sin prever que dicha idea de negocios traerán beneficios humanistas.

El objetivo de la banca tradicional, según establece la CONDUSEF (2016) es:"optimizar ganancias para sus accionistas". Este concepto establece dos posiciones, buscar ganancias y responder a accionistas, en ningún momento menciona la importancia del consumidor como pilar de la institución, ni prioriza la urgencia de los proyectos con enfoque social.

En este sentido, la banca tradicional se dirige a un sector general de la población, denominado beneficiario o cliente No obstante, deberán de cumplir ciertos parámetros o cualidades para ser acreedores de los servicios prestados por la institución financiera, y estos podrán variar dependiendo el producto que se busca contratar para financiarse.

La definición de financiamiento según BBVA (2021) es "proceso por el que se proporciona capital a una empresa o persona para utilizar en un proyecto o negocio, es decir, recursos como dinero y crédito para que pueda ejecutar sus planes"

Es así que se presume que el financiamiento es el medio que apoyará al comerciante a obtener distintos beneficios, ya sea buscar rentabilidad, capitalización, o expansión, para esto, el sistema financiero apoya con financiamiento crediticio. Dicho sistema, explica Álvarez (2020), se compone por la "banca de desarrollo, la banca comercial, las casas de bolsa, las sociedades de inversión, las arrendadoras y las aseguradoras, entre otros" (p.?)

Los productos financieros que manejan los bancos, se clasifican en dos: los de captación, los cuales buscan atraer recursos hacia el banco, tales como cuentas de depósito a la vista, pagarés e inversiones a plazos, estos prevén la obtención y resguardo de efectivo y sus equivalentes para el aprovechamiento de rendimientos por la institución. La segunda clasificación se divide en créditos, a través de los cuales se pueden obtener tarjetas de crédito, créditos hipotecarios, de nóminas y automotriz; además de otras opciones de inversión. estos productos generan un derecho a cobro por parte de la entidad hacia el cliente, obteniendo rendimientos sobre los montos prestados(Condusef, 2016).

Estos últimos son aquellos que el emprendedor busca para financiar su proyecto de negocio social, para solicitarlos debe contar con algunos requisitos, de los cuales según BBVA (2021) son:

1. Ser cliente del banco persona física o moral;

2. Contar con cuenta en el banco de débito o crédito;

3. Tener de 18 a 84 años de edad;

4. Que el tiempo del préstamo más la edad del cliente no supere 84 años con 364 días

5. Contar con buen historial crediticio y capacidad de pago.

6. Contar con INE

7. Comprobante de domicilio;

8. Estado de cuenta;

9. Comprobante de ingresos

En este sentido, se entiende que se debe un historial previo como referencia a la situación de deuda del emprendedor, además de que de esta manera se puede analizar su capacidad de pago.

No obstante, dentro de los mismos requisitos, se encuentran documentos limitantes para un nuevo emprendedor, como un buen historial crediticio, este sirve como herramienta para que el banco conozca su comportamiento ante las deudas; el no contar con uno, es un riesgo, sobre todo para el banco, debido a que no tiene referencia alguna sobre la liquidación de deuda ( Lanzagorta, 2021).

En este sentido, para poder acceder a un préstamo personal por el banco, será necesario contar con un historial. Sin embargo, ¿cómo pueden generar un historial si no les otorgan un crédito?

Esta es la problemática inicial de los negocios sociales, querer establecer un negocio, con la finalidad de cubrir una necesidad o una carencia social o ambiental, y estar limitado por no contar con un documento que les permita acceder a un financiamiento, lo cual es más común de lo que se cree. 
Las Micro, Pequeñas y Medianas Empresas (MIPyMES) expresaron a través de la Encuesta Nacional de Financiamiento (ENAFIN) aplicada por el Instituto Nacional de Estadística y Geografía (INEGI) que cuentan con menor acceso a financiamiento por parte de las instituciones financieras.

Además, sobresale información de descontento al obtener el financiamiento, ya que,el 54\% de los encuestados por INEGI (2019) expresó que los intereses que tendría que pagar, eran demasiado altos, el $23 \%$ que eran demasiados requisitos y documentos por cumplir, mientras que el resto estaba conforme.

Tabla 1. Estándar de rechazo de créditos

\begin{tabular}{l|c}
\hline \multicolumn{1}{c|}{ Motivo } & Media porcentual \\
\hline Sin historial crediticio & $27.8 \%$ \\
Baja capacidad de pago & $17.9 \%$ \\
$\begin{array}{l}\text { Documentación } \\
\text { insuficiente }\end{array}$ & $13.8 \%$ \\
$\begin{array}{l}\text { No le dijeron el motivo } \\
\text { Otros (No pudo } \\
\text { comprobar ingresos, Sin } \\
\text { garantía o aval, Mal } \\
\text { historial crediticio y }\end{array}$ & $19.6 \%$ \\
Sobreendeudamiento) & $20.9 \%$ \\
\hline \begin{tabular}{l} 
Total \\
\hline
\end{tabular} & $100 \%$ \\
\hline
\end{tabular}

Fuente: Elaboración Propia con datos de INEGI (2019)

Asimismo, INEGI menciona los factores más comunes por los que un financiamiento es negado por los bancos hacia los clientes, los cuales son falta de historial crediticio, baja capacidad de pago, documentación insuficiente, sin motivos y cuentan con un concepto denominados otros, que incluye no poder comprobar ingresos, no cuentan con garantía o aval, mal historial crediticio y sobre endeudamiento (INEGI, 2019).

Estos conceptos son los más comunes para rechazar el financiamiento a los clientes. En línea con lo anterior, en la Tabla 1, se puede observar que dentro de los cuales los más representativos, se encuentran la falta de historial crediticio con el $27.8 \%$ y el segundo es el concepto de otros, con el
$20.9 \%$ esto refleja las exigencias de los bancos tradicionales.

Tabla 1. Estándar de rechazo de créditos en México.

\begin{tabular}{|c|c|}
\hline Motivo & Media porcentual \\
\hline Sin historial crediticio & $27.8 \%$ \\
\hline Baja capacidad de pago & $17.9 \%$ \\
\hline Documentación insuficiente & $13.8 \%$ \\
\hline No le dijeron el motivo & $19.6 \%$ \\
\hline $\begin{array}{l}\text { Otros (No pudo comprobar } \\
\text { ingresos, Sin garantía o aval, } \\
\text { Mal historial crediticio y } \\
\text { Sobreendeudamiento) }\end{array}$ & $20.9 \%$ \\
\hline Total & $100 \%$ \\
\hline
\end{tabular}

No obstante, es importante identificar los porcentajes que afectan a cada uno de los tipos de empresas. Las MIPyMES en México se establecen por la cantidad de empleados según lo establece la Ley Para El Desarrollo de la Competitividad de la Micro, Pequeña y Mediana Empresa (LDCMIPYME, 2021).

Tabla 2.-Estratificación por Número de Trabajadores

\begin{tabular}{l|ccc}
\hline \multicolumn{1}{c}{ Sector/Tamaño } & \multicolumn{1}{c}{ Industria } & Comercio & \multicolumn{1}{c}{ Servicio } \\
\hline Micro & $0-10$ & $0-10$ & $0-10$ \\
Pequeña & $11-50$ & $11-30$ & $11-50$ \\
Mediana & $51-250$ & $31-100$ & $51-100$ \\
\hline
\end{tabular}

Como se puede mostrar en la Tabla 2, la asignación del tamaño de la empresa también se divide por sector en el que se desarrolla:industrial, comercial y de servicios. Una vez estratificadas se pueden analizar las motivaciones para cada tipo de sector. 
Tabla 2. Estratificación por Número de Trabajadores por tamaño de empresa en México

\begin{tabular}{l|ccc|}
\hline \multicolumn{1}{l}{ Sector/Tamaño } & \multicolumn{1}{c}{ Industria } & Comercio & \multicolumn{1}{c}{ Servicio } \\
\hline Micro & $0-10$ & $0-10$ & $0-10$ \\
Pequeña & $11-50$ & $11-30$ & $11-50$ \\
Mediana & $51-250$ & $31-100$ & $51-100$ \\
\multicolumn{2}{l}{ Fuente: LDCMIPYME (2021) }
\end{tabular}

Para las microempresas, según expresa INEGI (2019) el concepto más relevante para el rechazo del financiamiento es la falta de historial crediticio con el $47.3 \%$ lo cual, como comentaba Lanzagorta (2021) no contar con historial crediticio es perjudicial.

Para las pequeñas empresas el factor con mayor representatividad para el rechazo de financiamiento fue otros conceptos, con el 23.1\%; seguido por baja capacidad de pago con el $22.4 \%$, se consideran estos puntos debido a su similitud de porcentajes.

En el caso de las medianas empresas el $36.8 \%$ obtuvo una negativa de financiamiento al no cumplir con el concepto otros, similar al caso de las pequeñas empresas (INEGI, 2019).

Se puede observar que tanto la falta de historial crediticio y el no contar con un comprobante de ingresos suelen ser los principales problemas al momento de solicitar un préstamo.

Es así que, con las características recomendadas por el profesor Yunus sobre los créditos a baja tasa de interés y con la intención de apoyar a la sociedad y no reflejar la búsqueda de ganancias, lo cual, para la banca tradicional no es de interés.

Documentos como la falta de historial crediticio, en el que no es posible señalar que el cliente sea una persona morosa o incumplida con sus pagos, es más que suficiente para la banca tradicional, al no confiar en su honradez.

Esto, más allá de impedir la creación de un negocio, de una sola persona, atraería mayores consecuencias sociales, desde la insatisfacción de una necesidad socio-ambiental, la generación de empleos y la seguridad.
Conforme a lo establecido por la LDCMIPYME (2021) se puede suponer que, si la falta de crédito es el factor inicial para no emprender un negocio social. Para una pequeña empresa, significa que se perdió la oferta mínima de 11 puestos de trabajo, asimismo, entre 31 y 51 puestos de trabajo se pierden al no financiarse las medianas empresas.

La creación de empleos, ha sido un factor que a partir de la pandemia del COVID-19 ha sido difícil de mantener en marcha, así como la esperanza de mantener empleos es mínima, pero la creación de nuevos empleados es impensable.

El 15 de febrero del 2021, INEGI presentó los resultados de la Encuesta Nacional de Ocupación y Empleo (ENOE) en el que se comparó a la Población Económicamente Activa (PEA) del cuarto trimestre del 2019 con el del 2020.

Los resultados obtenidos de la ENOE reflejan un decremento en la PEA en comparación al ejercicio anterior(INEGI,2021).

Según cifras de INEGI (2021), dentro de la población ocupada, el $68.6 \%$ pertenece a trabajadores subordinados y remunerados, es decir, que de los 2.4 millones de empleos que se perdieron, 1.6464 millones pertenecían a este grupo.

La Ley Federal del Trabajo (LFT) en su artículo octavo, define como trabajador a toda "persona física que presta a otra, física o moral, un trabajo personal subordinado" (LFT, 2021).

En este sentido podemos observar en la Tabla 3, la presencia de disminución de trabajos subordinados y remunerados en un $68.6 \%$, hecho que implica otras preocupaciones, como la falta de servicios de salud social que se otorgan al trabajador subordinado, pausa temporal a las aportaciones para el retiro y él afore, dejando desprotegido al trabajador y sus familias.

Tabla 3. Comparativo de la Población Económicamente Activa en México. 2019-2020.

\begin{tabular}{l|ccc}
\hline \multicolumn{4}{c}{ Expresado en millones de personas } \\
\hline & 2019 & 2020 & Variación \\
\hline & & & \\
Población ocupada & 55.7 & 53.3 & -2.4 \\
\hline
\end{tabular}




\begin{tabular}{l|ccc}
\hline Mujeres & 22 & 20.7 & -1.3 \\
Hombres & 33.6 & 32.6 & -1.0 \\
\hline
\end{tabular}

Fuente: Elaboración propia con información de INEGI. (2021).

Además la LFT establece que para que exista un trabajador debe de existir un patrón, llámese negocio o empresa. Un porcentaje de despido de trabajadores significa menor capacidad de pago por poca obtención de ingresos.

En este sentido, la banca tradicional se verá afectada por la incapacidad de pago de empleadores y trabajadores, lo que a su vez acarrea menor capacidad de financiamiento a nuevos negocios que deseen emprender un proyecto.

De lo comentado anteriormente, aunado al estrés financiero, el cual según López (2014, p.6) se define como "la fuerza ejercida sobre los agentes económicos por la incertidumbre y las expectativas de los inversionistas sobre pérdidas en el valor de los activos financieros".

Este estrés fue determinado por el Banco de México (2020), el cual establece que a pesar de esperar una recuperación económica, la incertidumbre de los mercados financieros no disminuyen y es comparable junto al estrés financiero de 2009 que se presentó con la crisis económica global del 2008.

Adicionalmente, el Banco de México (2020) tomó en sus consideraciones finales, la importancia de dar seguimiento a los sectores que fueron mayormente afectados por la pandemia, aún teniendo en cuenta que la incertidumbre por los mercados persiste

Por último, el Banco de México (2020) contempla ciertos riesgos macrofinancieros los cuales son:una recuperación de la economía global menos vigorosa de lo anticipado, mayor volatilidad en los mercados financieros internacionales y una recomposición de flujos hacia activos de menor riesgo, una recuperación más lenta de la economía nacional $y$, ajustes en la calificación crediticia soberana y de Pemex.

\section{III.-MÉTODO.}

El presente trabajo se realizó con base en una investigación documental, derivada de la información recopilada de instituciones y autores, tales como Melé (2018) y Peruzzo (2020) quienes explican a la banca ética desde su nacimiento y su creación, y cómo se ha establecido en distintas partes del mundo.

Complementado con ordenamientos de carácter federal, como la Ley para el Desarrollo dea Competitividad de la Micro, Pequeña y Mediana Empresa y la Ley Federal de Trabajo, las cuales generaron definiciones de carácter legal.

Por último, cualitativamente también se consultaron intuiciones de banca comercial y banca ética. Instituciones éticas como Triodos Bank, Grameen Bank y Fiare Banca ética, quienes aportan información respecto al método de operar de la banca ética; comparado con instituciones comerciales como BBVA, quien aporta los requisitos de financiamiento y permite la comparación entre ambas bancas.

Asimismo, se considera una investigación de tipo mixta, la cual une la metodología cualitativa y cuantitativa. La recolección de datos cuantitativos fue obtenida de fuentes estadísticas como INEGI quien realizó la Encuesta Nacional de Financiamiento en las Empresas (ENAFIN) que incluye datos de créditos adquiridos, montos, motivos de rechazo, entre otros elementos Además del Banco de México, quien proyecta los riesgos financieros que se pueden presentar en distintas simulaciones (Reporte de Estabilidad Financiera).

Siguiendo con la revisión documental de INEGI, también se analizó la Encuesta Nacional de Ocupación y Empleo (ENOE) en la que se demuestra que hubo un decremento en el porcentaje de Población Económicamente Activa entre el año 2019 y 2020.

Por su parte, la investigación cualitativa surge de documentos y aportaciones de terceros, enfocados en el ámbito de la banca ética y tradicional.

Por lo que fue necesario consultar las leyes vigentes mexicanas en materia laboral y competitividad para esclarecer dicha información. 
En consecuencia, la investigación es de tipo comparativo, debido a que se centra en analizar las características del financiamiento tradicional y su impedimento en contraste a los estándares que propone la banca ética.

\section{IV.- RESULTADOS.}

\section{1.- Resultados de la investigación}

De la investigación documental realizada, se resuelve que la banca tradicional cuenta con estándares distintos al de la banca ética, lo cual imposibilita que negocios con objetivo social puedan emprender.

La dificultad de los trámites que presenta la banca tradicional para la aprobación de créditos también afecta a la creación de empleos, y al flujo de efectivo en las economías más vulnerables.

Es importante mencionar que el empleo conlleva a mayor recaudación a la federación, mediante contribuciones, e inclusive a las entidades federativas.

Dentro de los problemas que presentan las MIPyMES, sobre todo las microempresas, es la credibilidad, que depende de un historial crediticio al cual no pueden acceder por no contar con créditos.

Esto no permite cultivar la idea del profesor Muhammad Yunus, quien considera que proporcionando financiamiento se genera impacto social y económico, debido a que dichos financiamientos buscan ser de menor costo que las tasas tradicionales, y con base en la palabra del emprendedor, generando un nivel de confianza entre la entidad financiera y el negocio.

Esto demuestra que el modelo de Banca Ética, en México, sería de gran apoyo para el desarrollo sustentable de los negocios sociales, satisfaciendo necesidades de grupos vulnerables, generando empleos e ingresos por recaudación.

La Banca Ética supliría los servicios que la banca tradicional no está dispuesta a prestar por no ser negocios que le generan grandes utilidades para los socios, accionistas o inversores.

Esto demuestra que existe una verdadera necesidad de información y difusión de la banca ética y el emprendimiento social, para su implementación en México.

\subsection{Otros resultados obtenidos}

Cabe señalar que el emprendimiento cuenta con un alto nivel de aplicación potencial. de trabajadores que salieron de la PEA, necesitan cubrir sus obligaciones y necesidades, y a falta de empleo el emprendimiento es la solución legal más obvia.

Si bien, la intención de esta investigación no era medir la capacidad de demanda de la banca ética, el resultado obtenido es valorado para futuras investigaciones.

Por último también se identifica, que existe inconformidad por parte de los empresarios respecto a la banca tradicional; por lo que esto le permitirá a la Banca Ética establecerse y expandirse fácilmente en el territorio.

\section{CONCLUSIONES}

En conclusión, se observa que existe demanda para la banca ética, debido a los ideales que propone, no obstante el problema que presenta es que el concepto no es conocido entre el gremio empresarial, inclusive hace falta difundir su negocio social y su ideología.

El profesor Muhammad Yunus propone la idea de créditos a bajo costo, buscando el beneficio social antes que el económico, esto se contrapone a los objetivos que busca la banca tradicional, como lo expuso CONDUSEF en 2016.

De igual forma es necesario establecer, qué es un negocio social y sus características de forma legal, para poder constituir empresas con dicha denominación.

Asimismo, debería de existir una limitante para la banca con el modelo ético, apoyando exclusivamente a las empresas que realmente aporten un beneficio social, ambiental o ambos, y que permita el desarrollo económico de sus beneficiarios y colaboradores.

No obstante, se recomienda ser cuidadoso, si bien, la intención es confiar en la buena voluntad de las personas, tampoco se deberá financiar a todo tipo de negocios, se recomienda que exista un equipo 
especializado en evaluación de proyectos que realicen estudios de factibilidad.

Esto evitará hacer malas inversiones en entidades que son creadas sin un perfil social ni empresarial, haciendo ver las áreas de oportunidad al emprendedor para que vuelva una vez haya corregido los inconvenientes de su proyecto.

Sobre las garantías sobre el financiamiento, si bien, se busca perjudicar lo menos posible a los emprendedores que soliciten el financiamiento, es importante establecer una garantía, desde los activos adquiridos con el financiamiento, hasta un aval.

En este sentido también es recomendable considerar un seguimiento al desarrollo de la entidad financiada, no dejarlos solos y observar el comportamiento financiero y empresarial que lleva hasta el momento.

También es importante realizar un estudio sobre las dificultades que encontrará la banca ética al establecerse en México, desde cultura y política, que pueden ser totalmente distintas a los países que actualmente se encuentran establecidos.

Uno de los factores más importantes de reconocer es el salario mínimo mexicano, y los gastos que este acarrea consigo, contribuciones patronales a la seguridad, impuestos estatales, gastos de capacitación, entre otros.

Por lo que, deben reconocerse los salarios ofertados por la banca tradicional que oscilan entre los $\$ 8,000$ a los $\$ 14,000$ pesos en los puestos con menor requisitos, como cajero, asesor telefónico y asesor digital (INDEED, 2021).

Es así que se presume que los puestos de mayor requerimiento profesional, cuentan con salarios bastante elevados, la banca ética tendrá que observar si busca ser competitiva contra la banca tradicional con salarios similares, o no generar esa oferta.

Por último, el modelo de Banca Ética es ideal para el emprendimiento social, y la recuperación económica, no obstante existen algunos puntos a considerar, como los anteriormente mencionados, es así que este documento pretende servir como base para futuras investigaciones. 


\section{REFERENCIAS}

Álvarez, J. L. (2020, 26 marzo). ¿Qué es el sistema financiero y cómo se compone en México? elContribuyente.mx. https://www.elcontribuyente.mx/2019/05/\%F0\%9F\%92\%B5-que-es-el-sistema-financiero-y-como-se-compone-enmexico/

Banca Ética Latinoamericana. (2021). Banca Ética Latinoamericana - Sitio Web. bancaetica.lat. https://bancaetica.lat/

Banco de México. (2020). Reporte de Estabilidad Financiera - Segundo Semestre 2020. banxico.org.mx. https://www.banxico.org.mx/publicaciones-y-prensa/reportes-sobre-el-sistema-financiero/\%7BBB59C14C-03BE58EE-6E0F-7D3EB65D52D5\%7D.pdf

BBVA. (2021, 8 marzo). Financiamiento. bbva.mx. https://www.bbva.mx/educacion-financiera/f/financiamiento.html

BBVA. (2021). Préstamo Personal Inmediato | BBVA México. ww.bbva.mx. https://www.bbva.mx/personas/productos/creditos/prestamos-personales/prestamo-personal-inmediato.html

Cámara de diputados del h. congreso de la unión, (2021), Ley Federal del Trabajo, Diputados.gob.mx http://www.diputados.gob.mx/LeyesBiblio/pdf/247_130819.pdf

Cámara de diputados del h. congreso de la unión, (2021),Ley para el Desarrollo de la Competitividad de la micro, pequeña y mediana empresa.diputados.gob.mx,http://www.diputados.gob.mx/LeyesBiblio/pdf/247_130819.pdf

Cantuarias, S. (2021). A Latin American ethical bank as the heart of the economy and the creative industries. Creativity, Culture \& Capital. https://www.creativityculturecapital.org/a-latin-american-ethical-bank-as-the-heart-of-theeconomy-and-the-creative-industries/

Condusef. (2016). Condusef comparativo-banca. condusef.gob.mx. https://www.condusef.gob.mx/?p=comparativo-banca

Doble Impacto. (2021, 13 enero). ¿Qué es Doble Impacto? https://www.dobleimpacto.cl/que-es-doble-impacto/

Fiare Banca Ética. (2021). Nuestra historia. https://www.fiarebancaetica.coop/quienes-somos/nuestra-historia

Grameen Bank. (2021). Founder - Grameen Bank. Grameenbank.Org. https://grameenbank.org/founder-2/

INDEED. (2021). Bolsa de trabajo de Banco en Monterrey, N. L. - mayo 2021 | Indeed.com México. https://mx.indeed.com/trabajo?q=banco\&l=Monterrey,\%20N.\%20L.\&vjk=06782c56d1e06ab7

INEGI. (2019). Encuesta Nacional de Financiamiento de las Empresas 2018. https://www.inegi.org.mx/. https://www.inegi.org.mx/contenidos/programas/enafin/2018/doc/ENAFIN2018Pres.pdf

INEGI. (2021). Resultados de la Encuesta Nacional de Ocupación y Empleo 2020. inegi.org.mx. https://www.inegi.org.mx/contenidos/saladeprensa/boletines/2021/enoe_ie/enoe_ie2021_02.pdf

Lanzagorta, J. (2021). Tener un buen historial crediticio es mejor que no tener ninguno. El Economista. https://www.eleconomista.com.mx/opinion/Tener-un-buen-historial-crediticio-es-mejor-que-no-tener-ninguno20210210-0099.html

López, Y. (2014). Una medida de estrés financiero para México, su relación con la actividad económica y los mecanismos de transmisión del estrés. https://repositorio.tec.mx/. https://repositorio.tec.mx/bitstream/handle/11285/628891/33068001114637.pdf?sequence=1

Melé, J. A. (2018, 21 agosto). El origen de la banca ética. revista triodos. https://revista-triodos.com/articulos/2018/elorigen-de-la-banca-etica

Peruzzo, M. (2020). Banca ética: Definición y Características, 2016-2019. bdigital.uncu.edu.ar. https://bdigital.uncu.edu.ar/objetos_digitales/15714/banca-tica-definicin-y-caractersticas-2016-2019.pdf

Pineda, A. (2019). Yunus, el emprendedor social que ganó el Premio Nobel. Disruptivo.tv. https://disruptivo.tv/columnas-ynotas/yunus-el-profesor-que-gano-el-premio-nobel/

Triodos Bank UK. (2021). About us | Triodos Bank. triodos.co.uk. https://www.triodos.co.uk/about-us 